\title{
Slope and amplitude asymmetry effects on low frequency capacitively coupled carbon tetrafluoride plasmas
}

\author{
B. Bruneau, ${ }^{1, a)}$ I. Korolov, ${ }^{2}$ T. Lafleur, ${ }^{3}$ T. Gans, ${ }^{4}$ D. O'Connell, ${ }^{4}$ A. Greb, ${ }^{4}$ A. Derzsi, ${ }^{2}$ \\ Z. Donkó, ${ }^{2}$ S. Brandt, ${ }^{5}$ E. Schüngel, ${ }^{5}$ J. Schulze, ${ }^{5}$ E. Johnson, ${ }^{1}$ and J.-P. Booth ${ }^{3}$ \\ ${ }^{1}$ LPICM-CNRS, Ecole Polytechnique, 91128 Palaiseau, France \\ ${ }^{2}$ Institute for Solid State Physics and Optics, Wigner Research Centre for Physics, Hungarian Academy \\ of Sciences, Konkoly-Thege Miklós Str. 29-33, H-1121 Budapest, Hungary \\ ${ }^{3}$ LPP, Ecole Polytechnique-CNRS-Univ Paris-Sud-UPMC, 91128 Palaiseau, France \\ ${ }^{4}$ Department of Physics, York Plasma Institute, University of York, Heslington, York YO10 5DD, \\ United Kingdom \\ ${ }^{5}$ Department of Physics, West Virginia University, Morgantown, West Virginia 26506-6315, USA
}

(Received 12 February 2016; accepted 12 April 2016; published online 26 April 2016)

\begin{abstract}
We report investigations of capacitively coupled carbon tetrafluoride $\left(\mathrm{CF}_{4}\right)$ plasmas excited with tailored voltage waveforms containing up to five harmonics of a base frequency of $5.5 \mathrm{MHz}$. The impact of both the slope asymmetry, and the amplitude asymmetry, of these waveforms on the discharge is examined by combining experiments with particle-in-cell simulations. For all conditions studied herein, the discharge is shown to operate in the drift-ambipolar mode, where a comparatively large electric field in the plasma bulk (outside the sheaths) is the main mechanism for electron power absorption leading to ionization. We show that both types of waveform asymmetries strongly influence the ion energy at the electrodes, with the particularity of having the highest ion flux on the electrode where the lowest ion energy is observed. Even at the comparatively high pressure (600 mTorr) and low fundamental frequency of $5.5 \mathrm{MHz}$ used here, tailoring the voltage waveforms is shown to efficiently create an asymmetry of both the ion energy and the ion flux in geometrically symmetric reactors. Published by AIP Publishing.
\end{abstract}

[http://dx.doi.org/10.1063/1.4947453]

\section{INTRODUCTION}

Capacitively coupled radio frequency (CCRF) plasmas are commonly used in a wide range of applications, including thin film deposition and plasma etching, with end products in the semiconductor industry or in the medical sector. ${ }^{1}$ In the case of plasma etching, electronegative gases such as carbon tetrafluoride $\left(\mathrm{CF}_{4}\right)$ are generally used, ${ }^{2}$ often driven by a low frequency $^{3}$ of a few megahertz. These processes usually require control of the ion flux as well as the ion energy, a feature unachievable in classical single frequency CCRF discharges. ${ }^{4}$ It is possible, however, to control the ion energy using well-separated multiple frequencies or through frequency coupling of harmonics, for example, via the electrical asymmetry effect (EAE), independently of other plasma parameters and, in particular, independently of the ion flux. ${ }^{5-16}$ In this paper, we will investigate the effect of both amplitude asymmetric and slope asymmetric waveforms on ion properties in $\mathrm{CF}_{4}$ plasmas.

According to an analytical model developed by Czarnetzki et al., the appearance of a self-bias, $\eta,{ }^{5-7}$ due to the EAE, can be expressed as

$$
\eta=-\frac{\tilde{V}_{m 1}+\varepsilon \tilde{V}_{m 2}}{1+\varepsilon},
$$

\footnotetext{
a) Author to whom correspondence should be addressed. Electronic mail:
} bastien.bruneau@polytechnique.edu. where $\tilde{V}_{m 1}$ and $\tilde{V}_{m 2}$ are the maximum and the minimum (relative to the temporal average) of the applied voltage waveform $V_{A C}(t)$, respectively, and $\varepsilon$ is the symmetry parameter

$$
\varepsilon=\left|\frac{\hat{V}_{s g}}{\hat{V}_{s p}}\right| \approx\left(\frac{A_{p}}{A_{g}}\right)^{2}\left(\frac{Q_{m g}}{Q_{m p}}\right)^{2} \frac{\bar{n}_{s p}}{\bar{n}_{s g}},
$$

where $\hat{V}_{s p}$ and $\hat{V}_{s g}$ are the maximum sheath voltages across the sheaths at the powered and grounded electrodes, respectively; $A_{p}$ and $A_{g}$ are the area of the powered and the grounded electrodes; $Q_{m g}$ and $Q_{m p}$ are the maximum unbalanced charges in the respective sheaths; and $\bar{n}_{s p}$ and $\bar{n}_{s g}$ are the respective mean ion densities in the sheaths. Following the pioneering work of Wendt et al., ${ }^{17,18}$ waveforms comprising multiple harmonics of the same fundamental frequency, $f$, known as Tailored Voltage Waveforms (TVWs), have been shown to provide yet more control of the EAE compared to the two-frequency case. ${ }^{19}$ Such amplitude asymmetric waveforms are based on the following form for the driving voltage waveform: ${ }^{20}$

$$
V_{A C}(t)=V_{0} \sum_{k=1}^{N} \frac{N-k+1}{N} \cos (k \omega t+\Theta),
$$

where $V_{0}$ is a voltage amplitude factor, $N$ is the number of harmonics (here varied between one and five), $\omega=2 \pi f$ is the angular frequency (here corresponding to a frequency $f=5.5 \mathrm{MHz}$ ), and $\Theta$ is a phase shift. $V_{0}$ is set to give the 
desired peak-to-peak voltage, $V_{P P}$. The amplitudes of the individual harmonics are chosen to maximize the amplitude asymmetry of the waveform $\left(\tilde{V}_{m 1} / \tilde{V}_{m 2}\right)$ and, therefore, maximize the accessible range of the DC self-bias according to Eq. (1). ${ }^{20}$ The phase shift $\Theta$ can be varied to control the amplitude asymmetry from a maximum $(\Theta=0)$ to a minimum $(\Theta=\pi)$. Such waveforms, with $N=4$, are shown in Fig. 1(a) for different values of $\Theta$.

Alternatively, it was recently shown ${ }^{21-23}$ that some control over the asymmetry of argon discharges can be obtained using sawtooth waveforms (i.e., with differing rise and fall rates), defined by the following expression:

$$
V_{A C}(t)= \pm V_{0} \sum_{k=1}^{N} \frac{1}{k} \sin (k \omega t) .
$$

The slope asymmetry of the waveform can then be controlled by the number of frequencies, $N$. The $1 / k$ pre-factors are chosen to maximize the slope asymmetry for a given $N$. The minus sign in Eq. (4) corresponds to sawtooth-up waveforms, while the plus sign corresponds to sawtooth-down waveforms. Such waveforms, with different $N$, are shown in Fig. 1(b) (the case $N=1$ corresponds to a pure sinusoid). Here, the increase of the rising slope with $N$ can be clearly observed. These waveforms were shown to induce a fast sheath expansion and a slow sheath contraction in front of one electrode, and a slow sheath expansion and a fast sheath contraction on the other side. ${ }^{23}$ As a consequence, a strong asymmetry of the electron power absorption (and therefore
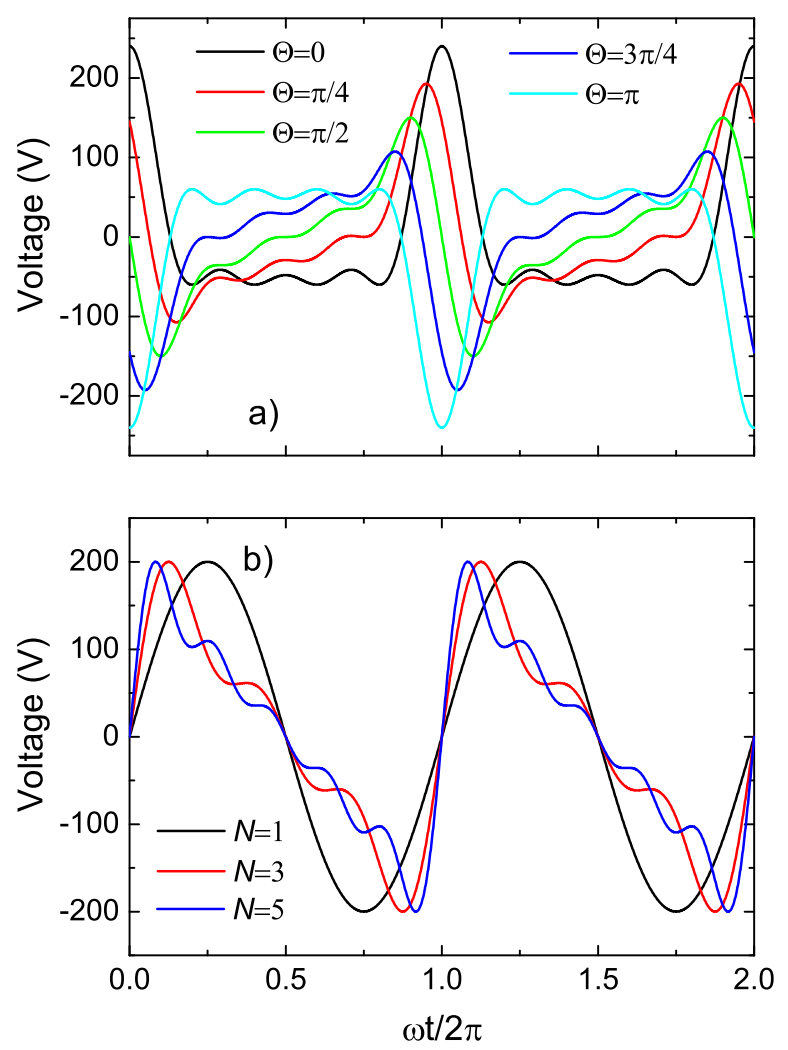

FIG. 1. (a) Waveforms according to Eq. (3) with different phase shifts, $\Theta$, and (b) waveforms according to Eq. (4), corresponding to sawtooth-down waveforms, for different numbers of harmonics $N$. the ionization rate) can be produced in geometrically symmetric argon discharges, with larger ionization closer to the electrode where the sheath expands rapidly. A DC self-bias was also shown to appear in argon discharges in this case, despite the absence of an amplitude asymmetry (i.e., $\tilde{V}_{m 1}=\tilde{V}_{m 2}$ ), because of different mean ion densities in the sheaths $(\varepsilon \neq 1$ in Eq. (1)), and therefore different ion fluxes at the electrodes.

More recently, Bruneau et al. ${ }^{24}$ have studied the impact of waveforms with continuously changing amplitude and slope asymmetry, obtained by slightly shifting the higher frequencies from the harmonics. These waveforms are shown to control the shape of the ion flux distribution function and, in particular, its width, while keeping the ion flux and mean ion energy constant. They are, however, not used in the present study.

Although the impact of the amplitude and slope asymmetry of a waveform has been studied in detail in argon discharges, ${ }^{6-12,21-23}$ only one article by Schulze et al. ${ }^{25}$ has looked at the effect of TVWs on $\mathrm{CF}_{4}$ discharges, and it is briefly studied in an article by Bruneau et al. ${ }^{26}$ The former study contained only simulations with no experimental measurements and was limited to two harmonics. Furthermore, there is an interest in studying the behavior of $\mathrm{CF}_{4}$ discharges when excited with low fundamental frequencies, as used in etching applications. ${ }^{3}$ In the present paper, the results of particle-in-cell (PIC) simulations are compared to experimental results, including phase-resolved optical emission spectroscopy (PROES) measurements. The impacts of both the amplitude and the slope of the waveform are investigated, using up to five harmonics with a fundamental frequency of $5.5 \mathrm{MHz}$.

\section{METHODS}

\section{A. Simulations}

The simulations consider four types of charged species: $\mathrm{CF}_{3}{ }^{+}, \mathrm{CF}_{3}{ }^{-}, \mathrm{F}^{-}$ions, and electrons. Although more species may be present, it has been shown in previous studies that these are the dominant ones. ${ }^{27-33}$ The energy-dependent cross sections for the electron- $\mathrm{CF}_{4}$ collision processes are presented in Fig. 2(a). The cross-sections are taken from Kurihara et al., ${ }^{34}$ except for electron attachment processes which are taken from Bonham et al. ${ }^{35}$ A complete list of the electron impact collision processes used in this model can be found in Schulze et al..$^{25}$ Although a large number of ion$\mathrm{CF}_{4}$ reactions take place in $\mathrm{CF}_{4}$ discharges, ${ }^{27,32,36}$ only a limited set of reactions are included here. The energy dependent cross sections for these reactions are shown in Fig. 2(b). These collision processes include reactive as well as elastic collisions of different ions with $\mathrm{CF}_{4}$. Because of the significant negative ion densities in these plasmas, recombination processes between positive and negative ions, as well as between electrons and $\mathrm{CF}_{3}{ }^{+}$, must be taken into account. These processes are simulated following the procedure by Nanbu and Denpoh. ${ }^{37}$ The rate of the electron- $\mathrm{CF}_{3}{ }^{+}$recombination is taken from Denpoh and Nanbu. ${ }^{38}$

The interelectrode distance is set to $2.5 \mathrm{~cm}$, and the neutral gas is assumed to be uniformly distributed with a 

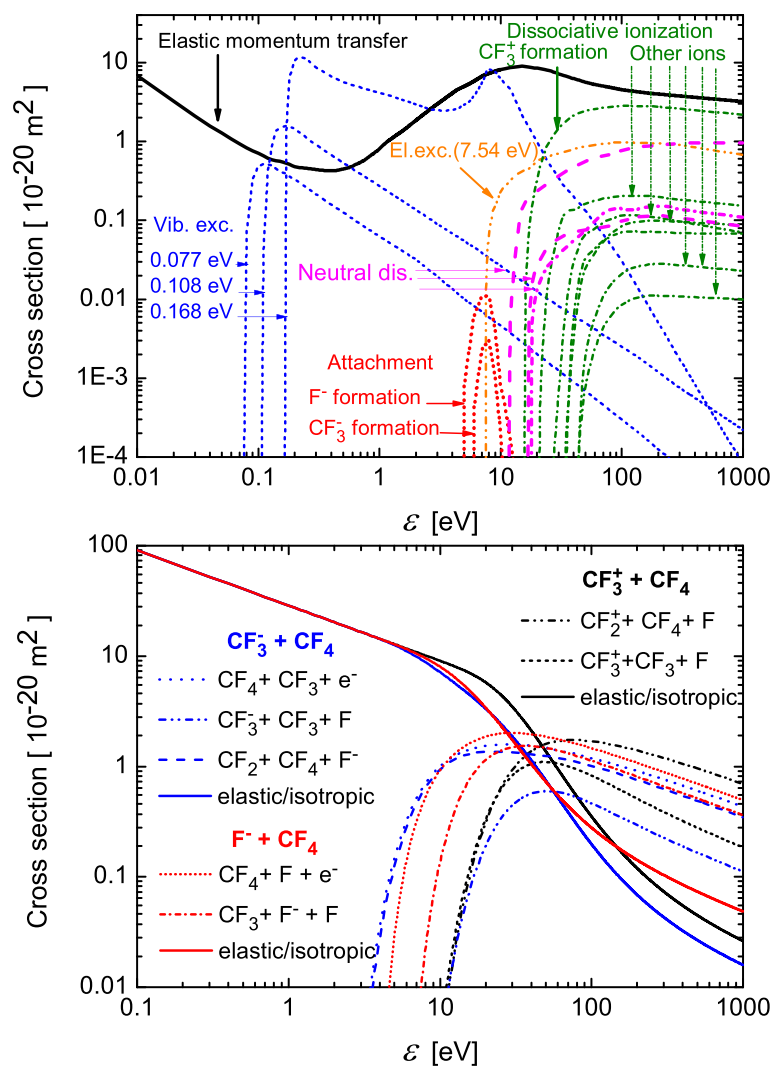

FIG. 2. Cross sections of electron-impact (a) and ion-impact (b) collision processes included in the simulations as a function of the electron and ion incident energy, respectively. Sources for the cross-sections are given in the text.

temperature of $350 \mathrm{~K}$. Electrons hitting the electrodes are reflected with a probability of $20 \%,{ }^{39}$ while the ion-induced emission of secondary electrons, $\gamma$, is set to 0 .

The DC self-bias in the simulations is determined as follows: at the beginning of the simulations, a bias of $0 \mathrm{~V}$ is set. After executing the simulation for $100 \mathrm{RF}$ cycles, the fluxes of the positively and negatively charged particles to each of the two electrodes are evaluated. Depending on the balance of these fluxes, the DC self-bias is changed by a small value. This procedure is continued until the DC selfbias reaches a converged value, and the charged particle fluxes to each of the two electrodes are balanced.

\section{B. Experiments}

The experimental setup has been described in detail in Ref. 23. A reactor with an inter-electrode gap of $2.5 \mathrm{~cm}$ was made geometrically symmetric by adding a thick Teflon ring (inner diameter $10 \mathrm{~cm}$ ), fitted with a $2.5 \times 2.5 \times 10 \mathrm{~cm}$ piece of borosilicate glass to allow optical access. The working pressure was varied between $50 \mathrm{mT}$ Torr and $600 \mathrm{mTorr}$ in the experiments. The voltage waveform was generated using a computer-controlled arbitrary function generator (AFG) and a broadband power amplifier as described previously. ${ }^{19}$ The true delivered waveform is monitored by a high voltage probe, and the waveform is corrected for distortion using a feedback loop as proposed by Patterson et al. ${ }^{18}$ Phaseresolved images of the plasma-induced optical emission are recorded with an intensified charge-coupled device (ICCD) camera (Andor iStar), which is synchronized to the AFG, and the phase varied using a delay generator. The gate time of the camera is $2 \mathrm{~ns}$, therefore giving 91 images within one RF-cycle for a fundamental frequency of 5.5 MHz. The excitation rate at each position and phase is derived from the measured emission from the $\mathrm{F}$ atom line at $703.7 \mathrm{~nm}$, using the deconvolution method proposed in Ref. 40, and is compared to the dissociative ionization rate from the simulations. The electron energy thresholds are $14.7 \mathrm{eV}$ (Ref. 41) for electron-impact excitation of the emitting state from ground state $\mathrm{F}$ atoms (experimentally observed) and $16 \mathrm{eV}$ for dissociative ionization (used in the simulations), respectively. Although two different processes are compared, these processes have similar energy thresholds and will therefore probe in a comparable way the high energy electron population. This strategy therefore limits us to a qualitative comparison of the dynamics of energetic electrons. A more thorough way of addressing this issue would be to include the $\mathrm{F}$ atom distribution in the simulations. Such a refinement, which remains for future work, may lead to the possibility of a more quantitative comparison between experiment and simulation.

\section{RESULTS}

In the following, the impact of waveforms with an amplitude asymmetry (according to Eq. (3)) on a $\mathrm{CF}_{4}$ discharge is investigated in Section III A, while in Section III B, we focus on the impact of waveforms with a slope asymmetry (according to Eq. (4)). In both cases, the excitation rates and the DC self-bias are compared between the simulations and the experiments, and the simulations are used to investigate the impact of TVWs on the ion flux and the ion flux-energy distribution function (IFEDF) at each electrode, since these could not be measured here.

\section{A. Amplitude asymmetry}

In this case, waveforms according to Eq. (3) are used, with $N=4$, and the phase shift $\Theta$ is varied to control the amplitude asymmetry of the waveform. The neutral gas pressure is kept constant at $600 \mathrm{mTorr}$, and $V_{0}$ is set to $96 \mathrm{~V}$ (giving a peak-to-peak voltage $V_{P P}=300 \mathrm{~V}$ for $\Theta=0$ ).

Figure 3 shows the DC self-bias, $\eta$, observed experimentally and given by the simulations as a function of $\Theta$. The agreement is good for almost all values of $\Theta$, except for $\Theta=0.75 \pi$ and $\Theta=1.75 \pi$. The DC self-bias voltage, $\eta$, increases as $\Theta$ is increased between 0 and $\pi$, and then decreases, consistent with the amplitude ratio $\tilde{V}_{m 1} / \tilde{V}_{m 2}$, which decreases in the first range and increases in the second one. It is worth noting that the variation of the DC self-bias with $\Theta$ is not as linear as in argon. ${ }^{21}$ Indeed, for $\Theta$ lower than $0.5 \pi$, it only slightly increases with $\Theta$, whereas it increases more significantly for $\Theta$ between $0.5 \pi$ and $\pi$. Overall, the range over which the DC self-bias can be controlled is significantly larger than that observed previously in the case of argon, ${ }^{10,12,20}$ or in $\mathrm{CF}_{4}{ }^{25}$ (although only two frequencies have been used in the latter case).

In the following, $\Theta$ is varied only between 0 and $\pi$, since for $\Theta$ between $\pi$ and $2 \pi$, the discharge is simply a 


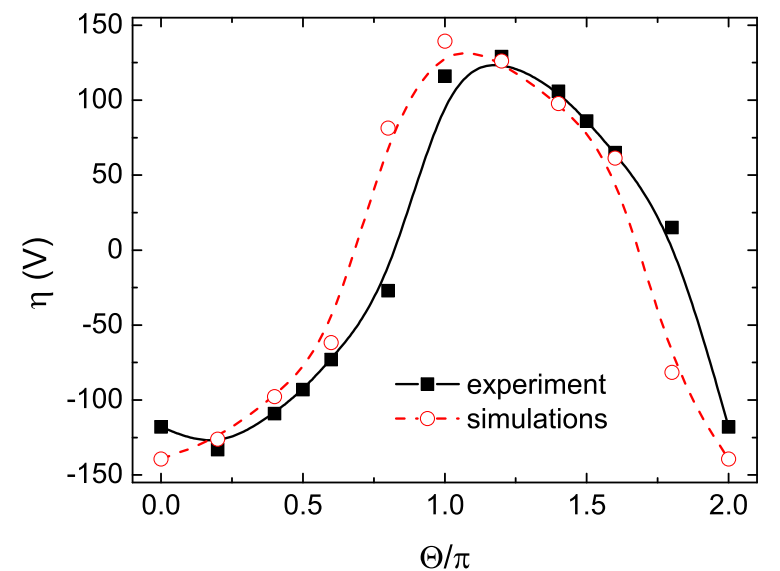

FIG. 3. DC self-bias, $\eta$, as a function of $\Theta$. The black solid line and squares represent the experimental results, and the red dashed line and circles represent the results of PIC simulations.

mirror image of that for $\Theta$ between 0 and $\pi$. Figure 4 shows the spatio-temporal dependence of the excitation rate, derived from the experimentally measured optical emission of the $\mathrm{F}$ line at $703.7 \mathrm{~nm}$ (top row), compared to the rate of $\mathrm{CF}_{4}$ dissociative ionization obtained from simulations (middle row), as a function of $\Theta$ from 0 to $\pi$ (from left to right). As mentioned in Section II, $\mathrm{F}$ atom excitation and $\mathrm{CF}_{4}$ dissociative ionization by electron impact have similar electron energy thresholds (of 14.5 and $16 \mathrm{eV}$, respectively), and should therefore have rates that vary in a similar way with electron density and temperature. The $x$-axis represents position (with the powered electrode at $x=0 \mathrm{~cm}$ and the grounded electrode at $x=2.5 \mathrm{~cm}$ ), while the $y$-axis represents time, and spans one fundamental RF cycle. The sheath edge position is obtained from the simulations using the Brinkmann criterion ${ }^{42}$ and is shown as a white line in the simulation plots. The images are normalized to the maximum of the excitation (or ionization) of the case at $\Theta=0.5 \pi$. Also shown in the bottom row is the dynamics of the electric field outside the sheath region (the latter being shown in black) obtained from the simulations.

The agreement between the experiments and the simulations is very good. The only significant difference is observed for $\Theta=0.75 \pi$, which is a region where the discharge asymmetry depends strongly on $\Theta$, as already observed in the DC self-bias voltage in Fig. 3. Therefore, a small difference in $\Theta$ between the experiments and the simulations will lead to large changes in the excitation dynamics. It should be noted that, in the experiments, a difference can be observed between the cases $\Theta=0$ and $\Theta=\pi$, whereas they should be mirror images of each other. This small discrepancy can be attributed either to imperfect optical alignment or to residual geometric asymmetry of the reactor.

Looking at the cases with $\Theta$ between 0 and $0.5 \pi$, one can see that the highest ionization peak occurs close to the grounded electrode during sheath contraction. This is the opposite of what has been observed for argon, ${ }^{9}$ where the highest ionization occurs at the sheath edge during sheath expansion. This can be explained by the different dominant electron power absorption mechanisms in $\mathrm{Ar}$ and $\mathrm{CF}_{4}$. In $\mathrm{Ar}$, electrons gain energy during the rf cycle predominantly by reflection from the expanding sheaths, whereas in $\mathrm{CF}_{4}$, electrons gain energy predominantly by the drift-ambipolar mechanism under many conditions, as described by Schulze et al. ${ }^{25,43}$ This can be observed in the bottom row of Fig. 4. Indeed, in this case, a strong electric field is present in the plasma bulk (outside the sheath). This field accelerates the electrons from the plasma centre towards the powered electrode. However, a floating potential sheath prevents these electrons from reaching the electrode, trapping them close to the electrode and creating a region with a high density of
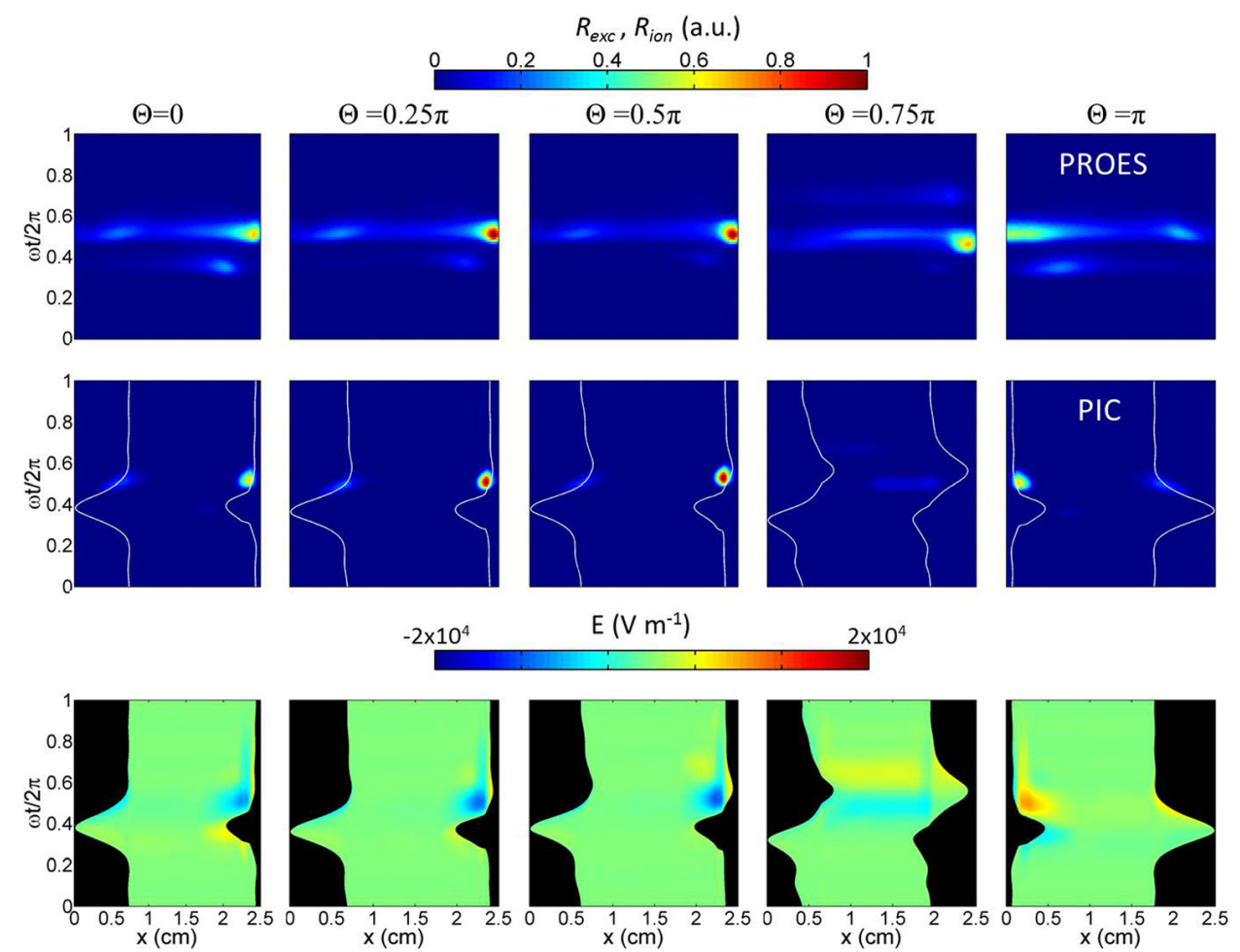

FIG. 4. Spatio-temporal excitation rate derived from measurements of the emission line at $703.7 \mathrm{~nm}$ using PROES (top row), rate of dissociative ionization (middle row), and electric field (bottom row) obtained from PIC simulations, using a waveform with $N=4$ harmonics according to Equation (3), for different values of $\Theta$ from 0 (left) to $\pi$ (right). The $x$-axis represents position (with the powered electrode at $x=0 \mathrm{~cm}$ and the grounded electrode at $x=2.5 \mathrm{~cm}$ ), while the $y$-axis represents time, and spans one fundamental RF cycle. 
highly energetic electrons, thus significantly increasing the rates of dissociative ionization and excitation. These two regions with electric fields in opposite directions can be regarded as a double layer, which develops in strongly electronegative gas discharges, even in single frequency excitation. ${ }^{44}$ The reasons for the development of such a structure in the case of multi-frequency excitation are studied in more detail in Ref. 45. The observed asymmetry therefore originates from the fact that this drift-ambipolar electric field only appears in front of a sheath which is rapidly collapsing.

Figure 5(a) shows the $\mathrm{CF}_{3}{ }^{+}$flux at the powered and grounded electrodes obtained from the simulations. For $\Theta=0$, the flux is highest at the grounded electrode, where the ion energy is lowest (as can be inferred from the large negative self-bias shown in Fig. 3 in this case). This is different from the case of argon, where the flux is highest at the electrode where the ion energy is highest. ${ }^{21}$ This is due, again, to the strong drift-ambipolar electric field which builds up in the plasma bulk in front of the grounded electrode sheath when it contracts. When $\Theta$ is increased to $\Theta=0.5 \pi$, the ion flux increases at the grounded electrode and decreases at the powered electrode, giving a flux 2.5 times larger at the grounded electrode compared to the
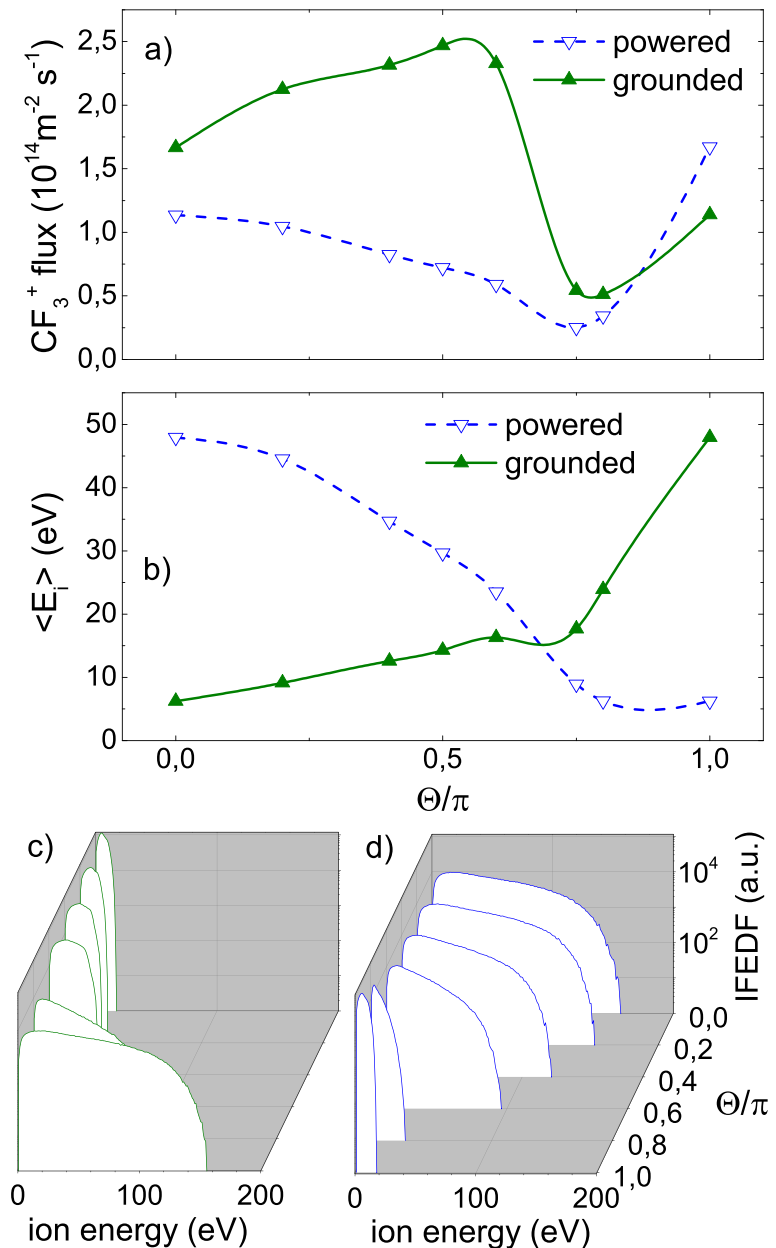

FIG. 5. Flux (a) and mean ion energy, $\left\langle E_{i}\right\rangle$, (b) of $\mathrm{CF}_{3}{ }^{+}$ions at the powered (blue dashed line and down-triangles) and grounded (green solid line and up-triangles) electrodes; $\mathrm{CF}_{3}{ }^{+}$flux-energy distribution function at the grounded (c) and powered (d) electrodes; these results have been obtained from the simulations. powered one. When $\Theta$ is further increased, the ion flux at the grounded electrode decreases strongly, reaching a minimum for $\Theta$ around $0.75 \pi$. This reduction is consistent with the lower ionization rate observed in Fig. 4, and with a significant change in the electric field dynamics.

Figure 5(b) shows the mean ion energy, $\left\langle E_{i}\right\rangle$, at the powered and grounded electrodes obtained from the simulations. For $\Theta=0$, the mean ion energy is close to $50 \mathrm{eV}$ at the powered electrode, and only about $6 \mathrm{eV}$ at the grounded electrode, i.e., the ratio of the mean energy values at the two electrodes is about eight. This result is surprising at such a high pressure, where the collisionality in the sheaths is high. This ratio is much larger than that obtained with two frequencies by Schulze et al. ${ }^{25}$ at a fundamental frequency of 13.56 MHz. The increase in the number of harmonics cannot explain this increase, and it must therefore be caused by the difference in the fundamental frequency. Such a study is, however, out of the scope of this paper and will be presented elsewhere. ${ }^{45}$ This result indicates that TVWs allow control of the ion energy over a large range in $\mathrm{CF}_{4}$ when the fundamental frequency is low, even at high pressures.

As a consequence of both the DC self-bias and the $\mathrm{CF}_{3}{ }^{+}$ flux dependence on $\Theta$, the ion flux-energy distribution function (IFEDF) evolution with $\Theta$ is non-trivial. The IFEDF, obtained from the simulations, is shown in Figs. 5(c) and $5(\mathrm{~d})$ for the grounded and powered electrodes, respectively. Note that the IFEDF is shown in log-scale. At the grounded electrode, the maximum ion energy remains low for $\Theta / \pi$ below 0.7 , which is consistent with the strong negative DC self-bias. Above this value, the maximum ion energy greatly increases, as a consequence of the increase of the DC selfbias, while the maximum of the IFEDF decreases, consistent with the reduction of the ion flux. The exact opposite occurs at the powered electrode.

\section{B. Slope asymmetry}

In the following, sawtooth waveforms according to Eq. (4) are used, and the number of harmonics composing the waveform $N$ is varied to control the slope asymmetry of the waveform. The fundamental frequency is set to $5.5 \mathrm{MHz}$, and the peak-to-peak voltage is $400 \mathrm{~V}$. In Ar, the strongest asymmetry was obtained at high pressures; ${ }^{22}$ therefore, we first examine the results at 600 mTorr. In addition, it was shown that the electronegativity of $\mathrm{CF}_{4}$ discharges increases with pressure. $^{25}$ Therefore, we investigated the effect of pressure on the discharge asymmetry over the range 50-600 mTorr.

\section{High pressure regime (600 mTorr)}

Figure 6(a) shows the DC self-bias obtained, when the discharge is excited with sawtooth-up and sawtooth-down waveforms. Full symbols and solid lines represent the experimental data, while the simulation results are represented by open symbols and dashed lines. The agreement between the simulations and the experiments is good, except for the case $N=2$, for which the simulations yield a significantly higher DC self-bias compared to the experiments. The reason for this discrepancy, despite the good agreement for the other cases, is still unclear. 

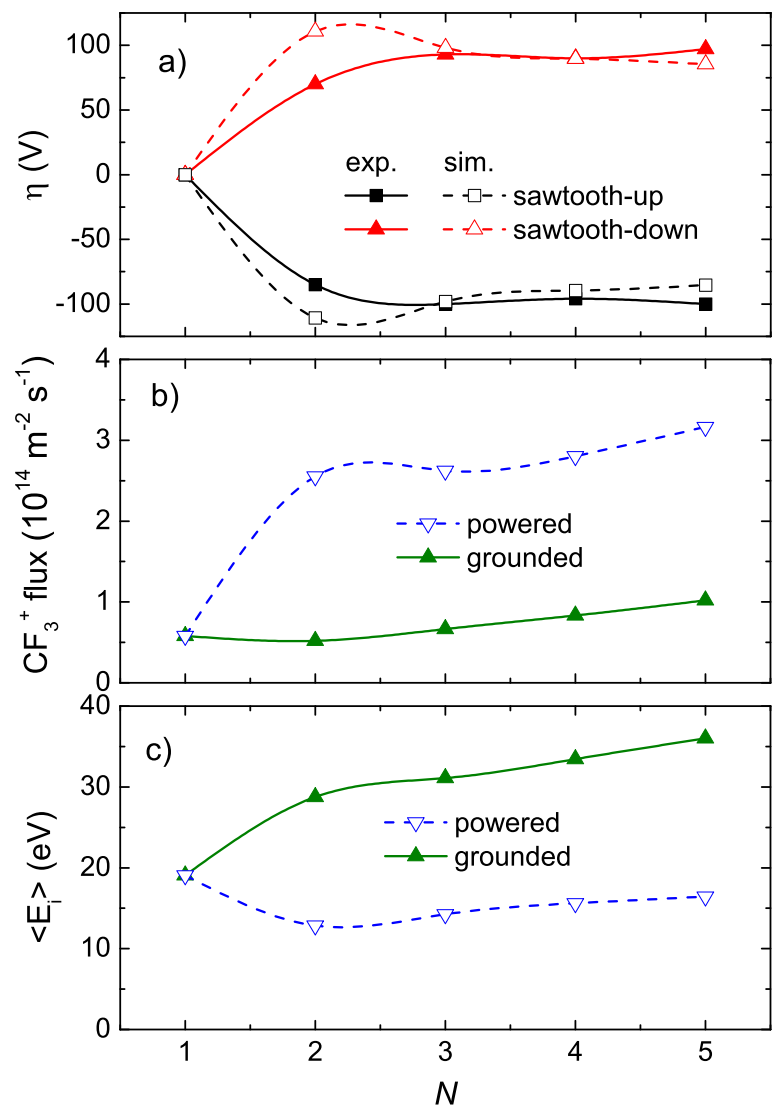

FIG. 6. (a) DC self-bias voltage, $\eta$, experimentally measured (solid lines and full symbols), and obtained from PIC simulations (dashed lines and open symbols), for sawtooth-up (black lines and up-triangles) and sawtooth-down (red lines and down-triangles) waveforms; (b) $\mathrm{CF}_{3}{ }^{+}$flux, at the powered (blue dashed line and down-triangles) and grounded (green solid line and up-triangles) electrodes, for a sawtooth-down waveform, obtained from the simulations; (c) mean ion energy, $\left\langle E_{i}\right\rangle, \mathrm{of}_{\mathrm{CF}_{3}}{ }^{+}$ions at the powered (blue dashed line and down-triangles) and grounded (green solid line and up-triangles) electrodes, for a sawtooth-down waveform, obtained from the simulations; these results are shown as a function of $N$.

The sawtooth-up waveforms lead to a negative DC selfbias, while the sawtooth-down waveforms give a positive one. This is the opposite of what was observed for Ar. ${ }^{22,23}$ In addition, the amplitude of the normalized DC self-bias can be more than two times higher than that obtained in Ar. Finally, whereas the DC self-bias continuously increases in argon as more harmonics are added, this is not the case for $\mathrm{CF}_{4}$ discharges, as no increase, or even a small decrease, is observed when increasing $N$ from 3 to 5 . The reason for this behavior lies in the $\mathrm{CF}_{3}{ }^{+}$flux to the electrodes. Indeed, as mentioned in the Introduction, whereas $\tilde{V}_{m 1}=\tilde{V}_{m 2}$ for these waveforms, the mean ion density at the sheath edges may differ, and so may the ion fluxes, leading to the appearance of a DC self-bias. We will see below that the ionization is highly asymmetric, leading to a large difference in the mean ion density in the two sheaths (e.g., $\bar{n}_{s p}>\bar{n}_{s g}$ ), leading to a symmetry parameter different from one according to Eq. (2) (e.g., $\varepsilon>1$ ) and, therefore, to a non-zero self-bias according to Eq. (1) (e.g., $\eta>0$ ).

Figure 6(b) shows the $\mathrm{CF}_{3}{ }^{+}$flux at the powered and grounded electrodes obtained from the simulations, for discharges excited with a sawtooth-down waveform. Whereas the ion flux at the grounded electrode increases only slightly with $N$, the ion flux at the powered electrode increases by a factor of 4.5 when $N$ is increased from 1 to 2 , and then continues to slowly increase when $N$ is further increased. As a consequence, the highest flux ratio of about 5 (with a higher flux at the powered electrode) is obtained for $N=2$. Therefore, the larger asymmetry in the normalized DC selfbias is linked to a larger asymmetry in the ion fluxes.

Figure 6(c) displays the mean ion energy, $\left\langle E_{i}\right\rangle$, at the powered and grounded electrodes obtained from the simulations. As one can see, and as expected from the positive DC self-bias, the ion energy is lower at the powered electrode. The ratio of the mean ion energy at both electrodes changes little as $N$ is varied, with a value of about 2.2. Therefore, for $N>1$, the ion flux is highest at the electrode where the ion energy is lowest.

In order to better understand how large the discharge asymmetry is at these conditions, Fig. 7 shows the simulated IFEDF at the powered and grounded electrodes with a sawtooth-down waveform for $N=2$ or $N=5$. Note that the vertical axis scale is logarithmic. As mentioned before, for $N=2$, the total ion flux obtained at the powered electrode is five times higher than at the grounded electrode. However, this figure also shows that the ion energy at the powered electrode is significantly lower, with a mean ion energy lower by a factor of two. Of interest is also the maximum ion energy, which was shown in Refs. 46 and 47 to be of great importance as far as material treatment is concerned, and which is four times lower at the powered electrode in the case $N=2$. For the case $N=5$, the asymmetry is slightly lower but remains very large (the ion flux is three times higher on the powered electrode, the mean ion energy is two times lower, and the maximum ion energy is three times lower).

Figure 8 shows the excitation rate extracted from $\mathrm{F}$ atom optical emission (top row), which is compared to the rate of dissociative ionization obtained from the simulations (second row), when the discharge is excited with a sawtooth-down waveform with $N=1$ to 5 (from left to right). The data for

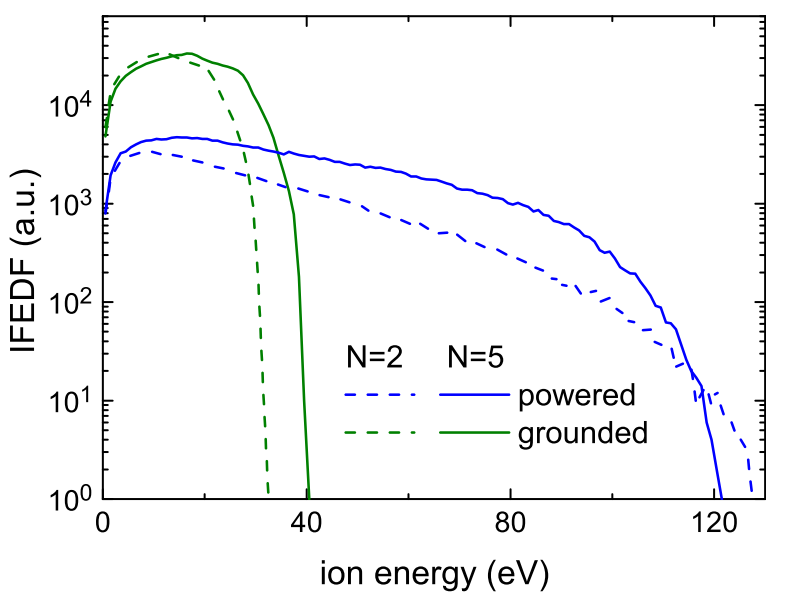

FIG. 7. Ion Flux-Energy Distribution Function (IFEDF) at the powered (blue lines) and grounded (green lines) electrodes for a sawtooth-down waveform with $N=2$ (dashed lines) or $N=5$ (solid lines), obtained from the simulations. 

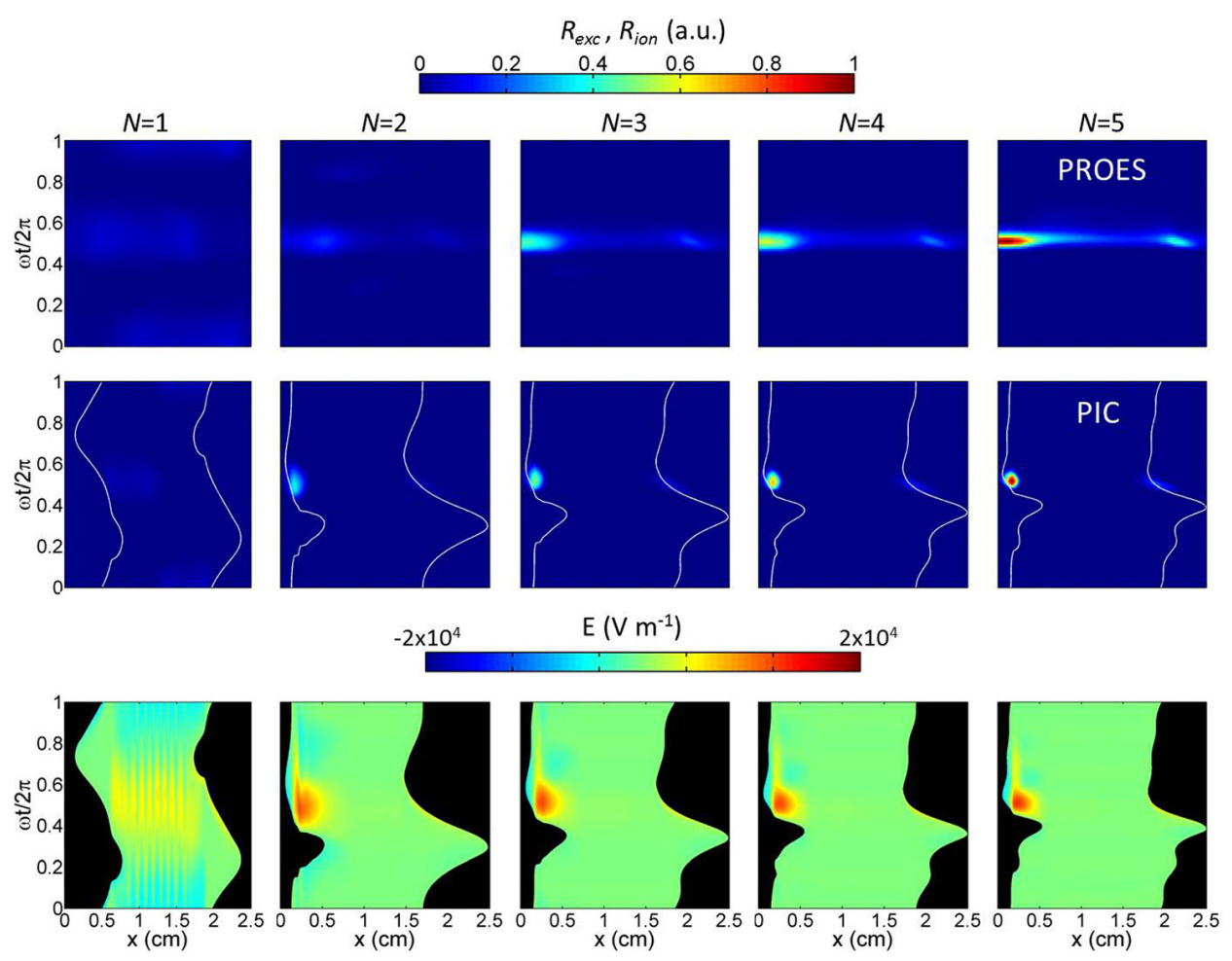

FIG. 8. Spatio-temporal excitation rate derived from measurement of the emission line at $703.7 \mathrm{~nm}$ using PROES (top row), the rate of dissociative ionization (middle row), and electric field (bottom row) obtained from PIC simulations, using a sawtoothdown waveform with $N=1$ to 5 (from left to right); the images are normalized by the maximum of the case with $N=5$.

both the simulations and the experiments are normalized to the maximum of the case $N=5$. The bottom row shows the dynamics of the electric field. The plots of the ionization rate also show the position of the edges of the sheaths. As $N$ is increased, the excitation strongly increases close to the powered electrode as the sheath collapses, while it increases less at the grounded electrode as that sheath expands. The excitation peak due to sheath expansion is slightly larger in the experiments (in particular, for large $N$ ), presumably due to a small optical misalignment (see also Fig. 4), that tends to decrease the amplitude close to the powered electrode.

This strong excitation close to the powered electrode indicates a significant difference in the excitation dynamics in $\mathrm{CF}_{4}$ plasmas compared to that observed in Ar. As shown in the bottom row of Fig. 8, this excitation occurs when a strong negative electric field appears close to this electrode, similar to what is observed in case of amplitude-asymmetric waveforms, indicating that the discharge is again operating in DA mode under these conditions. Once again, a double-layer structure is observed, ${ }^{44}$ which efficiently accelerates and traps electrons, leading to large excitation. The origin of the striations observed in the spatio-temporal electric field for the case $N=1$ is unknown. They were not observed experimentally, possibly because of limited spatial resolution.

In order to demonstrate the importance of the discharge asymmetry under these conditions, Fig. 9 shows (time-integrated) pictures through the PROES window of a $\mathrm{CF}_{4}$ discharge excited with a sawtooth-down waveform with $N=1$ (left) and $N=2$ (right). The arrows indicate the position of the powered (P.E.) and grounded (G.E.) electrodes. No postprocessing was applied to these images, i.e., they correspond to what can be seen by the naked eye. The discharge is observed in the central square, corresponding to the window in the Teflon ring. Whereas the light emission is uniform across the electrode gap for sinusoidal excitation $(N=1)$, it is strongly localized close to the powered electrode for a
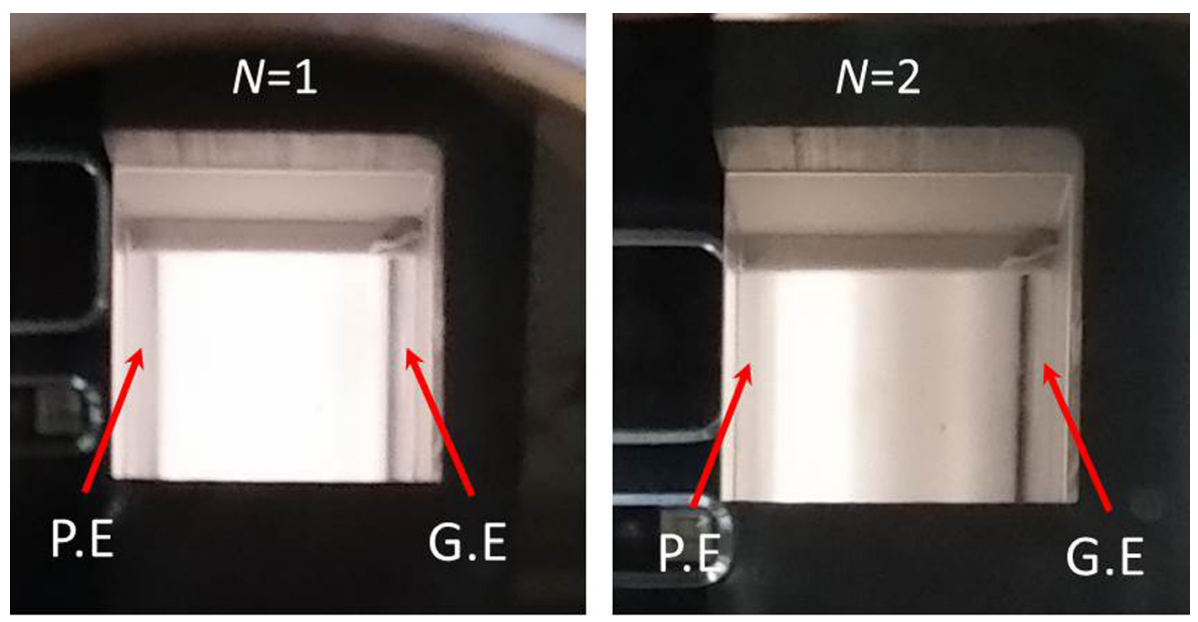

FIG. 9. Images taken through the PROES window of the $\mathrm{CF}_{4}$ discharge excited with a sawtooth-down waveform with $N=1$ (left) and $N=2$ (right). The arrows indicate the position of the powered (P.E.) and grounded (G.E.) electrodes. 
sawtooth waveform $(N=2)$; the emission of almost one half of the discharge has been "turned off." This is consistent with the PROES results shown in Fig. 2. Although not quantitative, these pictures give a visual impression of the strong impact of sawtooth waveforms on the spatial distribution of the discharge.

\section{Effect of gas pressure}

In this section, we explore how the effect of slope asymmetry depends on gas pressure. The other conditions are kept identical to those defined beforehand. It was shown previously that the gas pressure has a strong impact on the discharge asymmetry obtained with sawtooth waveforms in argon. ${ }^{22}$ Reducing the gas pressure increases the mean free path for energetic electrons, and this leads to changes to the spatial profiles of excitation and ionization. In addition, Schulze et al..$^{43}$ demonstrated that the impact of the DA heating decreases at lower pressure, which could also affect the discharge asymmetry.

Figure 10 shows the DC self-bias voltage, experimentally measured, and obtained from the PIC simulations, as a function of pressure $p$, for sawtooth-up and sawtooth-down waveforms, for (a) $N=2$, (b) $N=3$, and (c) $N=5$. The experimental trends are well reproduced by the simulations for each value of $N$.

In all cases, the DC self-bias voltage increases with pressure, similar to argon. ${ }^{22}$ For $N=2$, the DC self-bias increases linearly with pressure over the range investigated (both in the experiments and in the simulations), whereas for $N>2$, a strong increase of the DC self-bias is observed at a pressure of about 200 mTorr, followed by a weaker increase when pressure is further increased.

In order to understand the different behaviour for $N=2$ and $N>2$, Fig. 11 shows the rate of the dissociative ionization obtained from the simulations, for discharges excited with sawtooth-down waveforms with $N=2$ (first row) and $N=5$ (second row) for pressures of $50 \mathrm{mTorr}, 150 \mathrm{mTorr}$, 300 mTorr, and 600 mTorr (from left to right). No normalization has been applied to these images.

It can be seen that for $N=2$, the excitation remains close to the powered electrode over this pressure range. Increasing the pressure only makes the excitation features more localized and draws them closer to the powered electrode, and no clear transition can be observed. In contrast, for $N=5$, at low pressure the maximum excitation is closer to the grounded electrode, possibly because electron energy gain from sheath expansion is comparable to or larger than the energy gain from the drift-ambipolar electric field. However, when the pressure is increased to 300 mTorr, a sharp transition occurs and the excitation becomes strongly localized close to the powered electrode, possibly because the driftambipolar electric field becomes the main source for electron energy gain. The fact that this transition occurs for $N=5$ but not for $N=2$ might be due to the fact that the energy gain by sheath expansion strongly depends on $N$, as it was observed in argon. ${ }^{23}$ Therefore, the changes in the excitation dynamics explain why the DC self-bias shows a transition for $N>2$ but not for $N=2$.
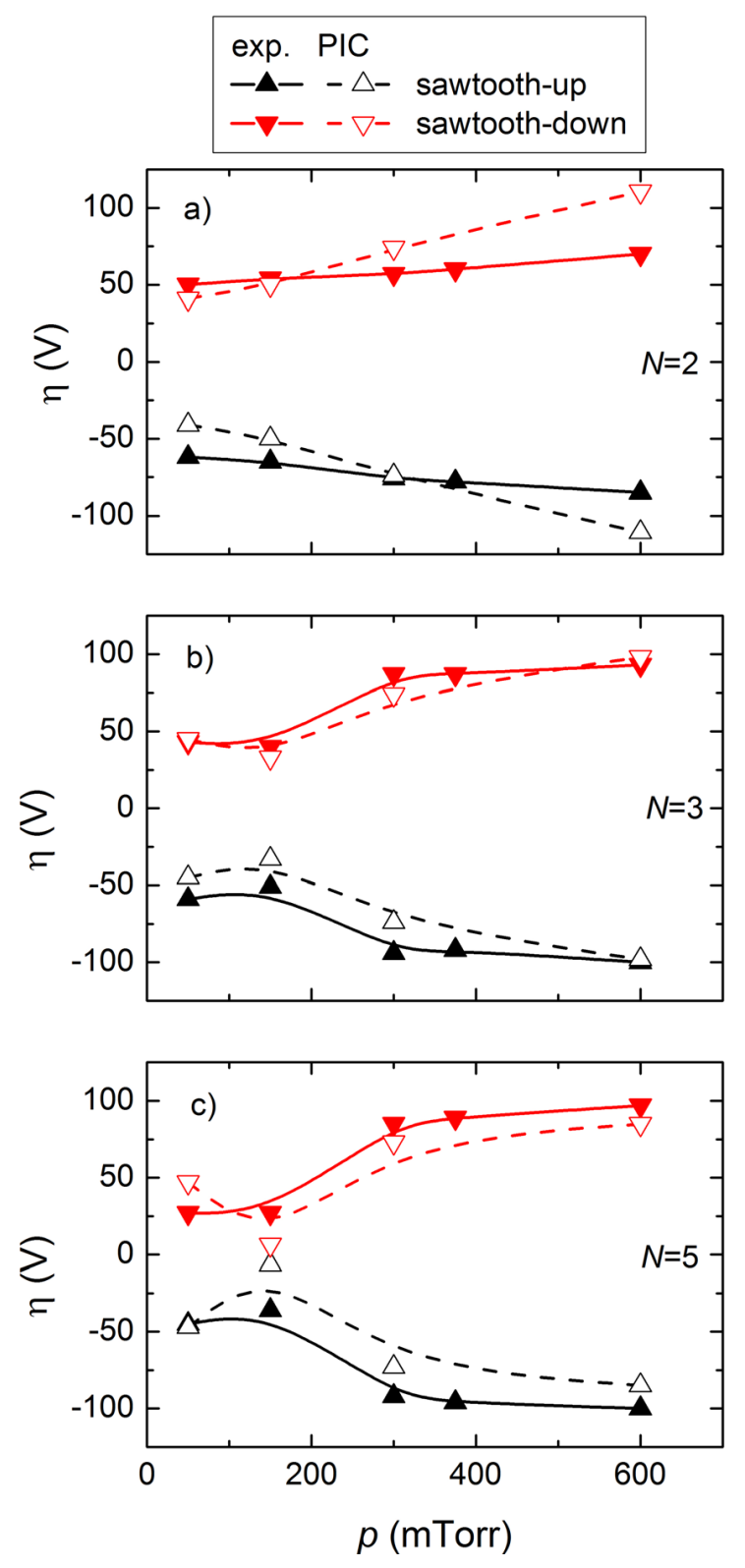

FIG. 10. DC self-bias voltage, experimentally measured (solid lines and full symbols), and obtained from PIC simulations (dashed lines and open symbols), as a function of pressure $p$, for sawtooth-up (black lines and up-triangles) and sawtooth-down (red lines and down-triangles) waveforms, for (a) $N=2$, (b) $N=3$, and (c) $N=5$.

\section{CONCLUSION}

We have investigated the impact of amplitude and slope asymmetry of the driving voltage waveforms on the electrical asymmetry of geometrically symmetric $\mathrm{CF}_{4}$ discharges. For all the conditions studied here, the discharge was found to operate in the DA mode, where a strong drift-ambipolar electric field adjacent to (but outside) the collapsing sheath is responsible for most of the ionization. Predictions of PIC simulations were found to be in good qualitative agreement with experimental data.

Controlling the amplitude asymmetry of the waveform via the phase shift $\Theta$ has been shown to be an efficient way of controlling the ion energy, with maximum energies up to nine times higher at one electrode compared to the other. However, at these high pressure conditions, significant 


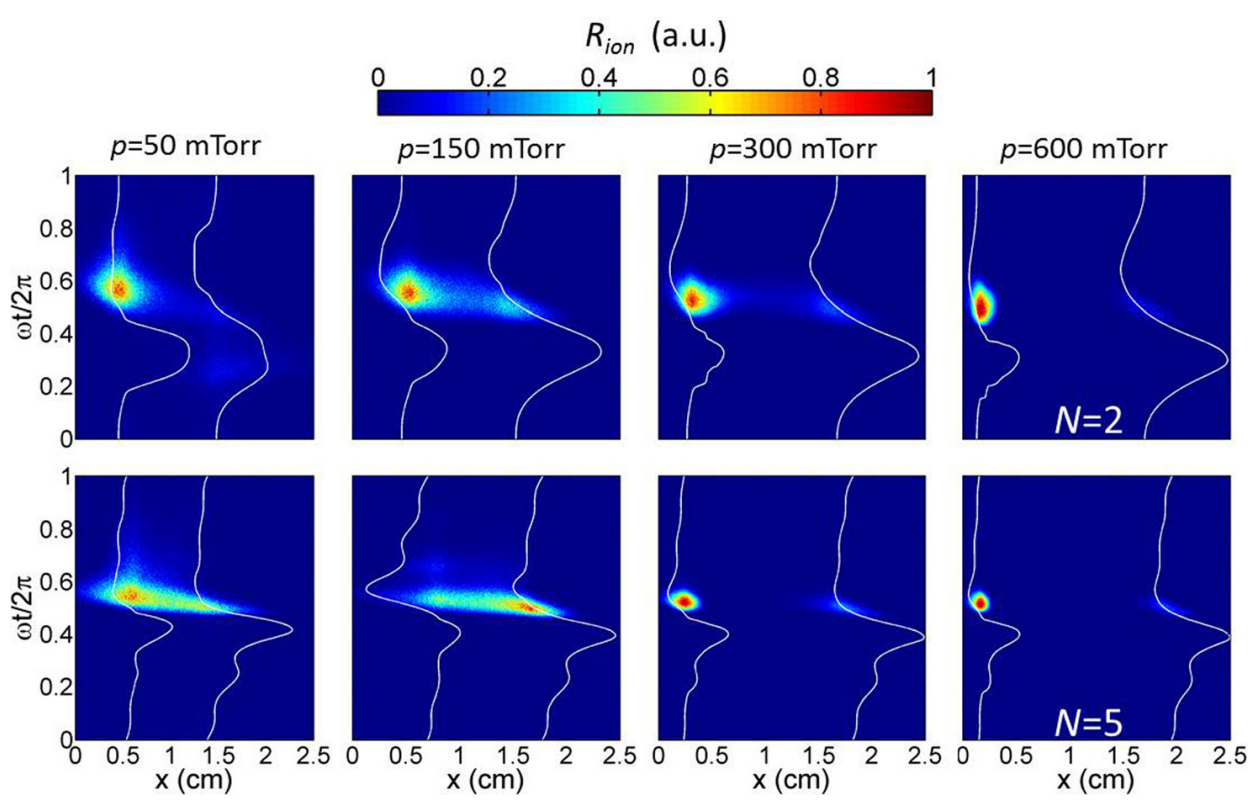

FIG. 11. Rate of dissociative ionization obtained from the simulations for a discharge excited with a sawtoothdown waveform for $N=2$ (first row) and $N=5$ (second row) for pressures of $50 \mathrm{mTorr}, 150 \mathrm{mTorr}, 300 \mathrm{mTorr}$, and $600 \mathrm{mTorr}$ (from left to right).

changes in the ion flux also occur as $\Theta$ is varied. In particular, much lower ion fluxes are observed specifically for $\Theta$ around $0.75 \pi$, where the effects of the slope asymmetry and the amplitude asymmetry of the waveform balance each other out.

This effect in $\mathrm{CF}_{4}$ occurs because, unlike for argon, the slope asymmetry of the waveform creates an ionization asymmetry as large as that created by the amplitude asymmetry. For instance, for some conditions, one electrode can have an ion flux five times higher than at the opposite electrode, while at the same time having a maximum ion energy four times lower. At lower pressures, electron energy gain via sheath expansion is more significant and can become comparable to that due to drift-ambipolar fields, therefore reducing the asymmetry.

To conclude, TVWs can be used to create a strong asymmetry of both the ion energy and the ion flux, even in geometrically symmetric reactors and with a low fundamental frequency. Larger (and inverted) effects are observed in electronegative gases $\left(\mathrm{CF}_{4}\right)$ compared to Ar, especially at higher pressure. This effect may be of potential interest for plasma etching applications.

\section{ACKNOWLEDGMENTS}

The authors would like to thank F. Farci and P. Bulkin for their help with the reactor used in this study. Work described in this paper was made possible by financial support from the ANR projects APOCALYPSO (ANR-13-PRGE-0003-01), CleanGRAPH (ANR-13-BS09-0019), the UK EPSRC (EP/ $\mathrm{K} 018388 / 1$ \& EP/H003797/1), the York-Paris Low Temperature Plasma Collaborative Research Centre, and the Hungarian Fund for Scientific Research (OTKA K 105476). B. Bruneau was also supported by the Doctoral School of Ecole Polytechnique (EDX grant, Ecole Doctorale de l'X).

${ }^{1}$ M. A. Lieberman and A. J. Lichtenberg, Principles of Plasma Discharges and Materials Processing (Wiley, 2005).

${ }^{2}$ D. L. Flamm and V. M. Donnelly, "The design of plasma etchants," Plasma Chem. Plasma Process. 1(4), 317-363 (1981).
${ }^{3}$ V. M. Donnelly, D. L. Flamm, W. C. Dautremont-Smith, and D. J Werder, "Anisotropic etching of $\mathrm{SiO}_{2}$ in low-frequency $\mathrm{CF}_{4} / \mathrm{O}_{2}$ and $\mathrm{NF}_{3} /$ Ar plasmas," J. Appl. Phys. 55(1), 242-252 (1984).

${ }^{4}$ A. Perret, P. Chabert, J. Jolly, and J.-P. Booth, "Ion energy uniformity in high-frequency capacitive discharges," Appl. Phys. Lett. 86(2), 021501 (2005).

${ }^{5}$ B. G. Heil, U. Czarnetzki, R. P. Brinkmann, and T. Mussenbrock, "On the possibility of making a geometrically symmetric RF-CCP discharge electrically asymmetric,” J. Phys. D: Appl. Phys. 41(16), 165202 (2008).

${ }^{6}$ J. Schulze, E. Schüngel, U. Czarnetzki, and Z. Donkó, "Optimization of the electrical asymmetry effect in dual-frequency capacitively coupled radio frequency discharges: Experiment, simulation, and model," J. Appl. Phys. 106(6), 063307 (2009).

7Z. Donkó, J. Schulze, B. G. Heil, and U. Czarnetzki, "PIC simulations of the separate control of ion flux and energy in CCRF discharges via the electrical asymmetry effect," J. Phys. D: Appl. Phys. 42(2), 025205 (2009).

${ }^{8}$ I. Korolov, Z. Donkó, U. Czarnetzki, and J. Schulze, "The effect of the driving frequencies on the electrical asymmetry of dual-frequency capacitively coupled plasmas," J. Phys. D: Appl. Phys. 45(46), 465205 (2012).

${ }^{9}$ T. Lafleur and J. P. Booth, "Control of the ion flux and ion energy in CCP discharges using non-sinusoidal voltage waveforms," J. Phys. D: Appl. Phys. 45(39), 395203 (2012).

${ }^{10}$ T. Lafleur, P. A. Delattre, E. V. Johnson, and J. P. Booth, "Separate control of the ion flux and ion energy in capacitively coupled radio-frequency discharges using voltage waveform tailoring," Appl. Phys. Lett. 101(12), 124104 (2012).

${ }^{11}$ T. Lafleur, R. W. Boswell, and J. P. Booth, "Enhanced sheath heating in capacitively coupled discharges due to non-sinusoidal voltage waveforms," Appl. Phys. Lett. 100(19), 194101 (2012).

${ }^{12}$ P.-A. Delattre, T. Lafleur, E. Johnson, and J.-P. Booth, "Radio-frequency capacitively coupled plasmas excited by tailored voltage waveforms: Comparison of experiment and particle-in-cell simulations," J. Phys. D: Appl. Phys. 46(23), 235201 (2013).

${ }^{13}$ D. J. Coumou, D. H. Clark, T. Kummerer, M. Hopkins, D. Sullivan, and S. Shannon, "Ion energy distribution skew control using phase-locked harmonic RF bias drive," IEEE Trans. Plasma Sci. 42(7), 1880-1893 (2014).

${ }^{14}$ Y. Zhang, A. Zafar, D. J. Coumou, S. C. Shannon, and M. J. Kushner, "Control of ion energy distributions using phase shifting in multifrequency capacitively coupled plasmas," J. Appl. Phys. 117(23), 233302 (2015).

${ }^{15}$ A. R. Gibson, A. Greb, W. G. Graham, and T. Gans, "Tailoring the nonlinear frequency coupling between odd harmonics for the optimisation of charged particle dynamics in capacitively coupled oxygen plasmas," Appl. Phys. Lett. 106(5), 054102 (2015).

${ }^{16}$ D. O'Connell, T. Gans, E. Semmler, and P. Awakowicz, "The role of the relative voltage and phase for frequency coupling in a dual-frequency capacitively coupled plasma," Appl. Phys. Lett. 93(8), 081502 (2008).

${ }^{17}$ S.-B. Wang and A. E. Wendt, "Control of ion energy distribution at substrates during plasma processing," J. Appl. Phys. 88(2), 643-646 (2000). 
${ }^{18}$ M. M. Patterson, H.-Y. Chu, and A. E. Wendt, "Arbitrary substrate voltage wave forms for manipulating energy distribution of bombarding ions during plasma processing," Plasma Sources Sci. Technol. 16(2), 257-264 (2007).

${ }^{19}$ E. V. Johnson, T. Verbeke, J.-C. Vanel, and J.-P. Booth, "Nanocrystalline silicon film growth morphology control through RF waveform tailoring," J. Phys. D: Appl. Phys. 43(41), 412001 (2010).

${ }^{20}$ J. Schulze, E. Schüngel, Z. Donkó, and U. Czarnetzki, “The electrical asymmetry effect in multi-frequency capacitively coupled radio frequency discharges," Plasma Sources Sci. Technol. 20(1), 015017 (2011).

${ }^{21}$ B. Bruneau, T. Novikova, T. Lafleur, J. P. Booth, and E. V. Johnson, "Ion flux asymmetry in radiofrequency capacitively-coupled plasmas excited by sawtooth-like waveforms," Plasma Sources Sci. Technol. 23(6), 065010 (2014).

${ }^{22}$ B. Bruneau, T. Novikova, T. Lafleur, J. P. Booth, and E. V. Johnson, "Control and optimization of the slope asymmetry effect in tailored voltage waveforms for capacitively coupled plasmas," Plasma Sources Sci. Technol. 24(1), 15021 (2015).

${ }^{23}$ B. Bruneau, T. Gans, D. O'Connell, A. Greb, E. V. Johnson, and J.-P. Booth, "Strong ionization asymmetry in a geometrically symmetric radio frequency capacitively coupled plasma induced by sawtooth voltage waveforms," Phys. Rev. Lett. 114(12), 125002 (2015).

${ }^{24}$ B. Bruneau, T. Lafleur, J.-P. Booth, and E. Johnson, "Controlling the shape of the ion energy distribution at constant ion flux and constant mean ion energy with tailored voltage waveforms," Plasma Sources Sci. Technol. 25(2), 025006 (2016).

${ }^{25}$ J. Schulze, A. Derzsi, and Z. Donkó, "Electron heating and the electrical asymmetry effect in dual-frequency capacitive CF 4 discharges," Plasma Sources Sci. Technol. 20(4), 045008 (2011).

${ }^{26}$ B. Bruneau, T. Lafleur, T. Gans, D. O'Connell, A. Greb, I. Korolov, A. Derzsi, Z. Donkó, S. Brandt, E. Schüngel, J Schulze, P. Diomede, D. J. Economou, S. Longo, E. Johnson, and J.-P. Booth, "Effect of gas properties on the dynamics of the electrical slope asymmetry effect in capacitive plasmas: Comparison of $\mathrm{Ar}, \mathrm{H}_{2}$ and $\mathrm{CF}_{4}$," Plasma Sources Sci. Technol. 25(1), 01LT02 (2016)

${ }^{27}$ V. Georgieva, A. Bogaerts, and R. Gijbels, "Numerical study of Ar/CF $/$ $\mathrm{N}_{2}$ discharges in single- and dual-frequency capacitively coupled plasma reactors," J. Appl. Phys. 94(6), 3748-3756 (2003).

${ }^{28}$ V. Georgieva, A. Bogaerts, and R. Gijbels, "Numerical investigation of ion-energy-distribution functions in single and dual frequency capacitively coupled plasma reactors," Phys. Rev. E 69(2), 026406 (2004).

${ }^{29}$ V. Georgieva and A. Bogaerts, "Numerical simulation of dual frequency etching reactors: Influence of the external process parameters on the plasma characteristics," J. Appl. Phys. 98(2), 023308 (2005).

${ }^{30} \mathrm{~V}$. Georgieva and A. Bogaerts, "Plasma characteristics of an $\mathrm{Ar} / \mathrm{CF}_{4} / \mathrm{N}_{2}$ discharge in an asymmetric dual frequency reactor: Numerical investigation by a PIC/MC model," Plasma Sources Sci. Technol. 15(3), 368 (2006).

${ }^{31}$ Z. Donkó and Z. L. Petrović, "Analysis of a capacitively coupled dualfrequency $\mathrm{CF}_{4}$ discharge," Jpn. J. Appl. Phys. Part 1 45(10S), 8151 (2006).
${ }^{32}$ K. Denpoh and K. Nanbu, "Self-consistent particle simulation of radiofrequency $\mathrm{CF}_{4}$ discharge with implementation of all ion-neutral reactive collisions," J. Vac. Sci. Technol. A 16(3), 1201-1206 (1998).

${ }^{33}$ O. V. Proshina, T. V. Rakhimova, A. T. Rakhimov, and D. G. Voloshin, "Two modes of capacitively coupled rf discharge in $\mathrm{CF}_{4}$," Plasma Sources Sci. Technol. 19(6), 065013 (2010).

${ }^{34}$ M. Kurihara, Z. L. Petrovic, and T. Makabe, "Transport coefficients and scattering cross-sections for plasma modelling in $\mathrm{CF}_{4}$-Ar mixtures: A swarm analysis," J. Phys. D: Appl. Phys. 33(17), 2146 (2000).

${ }^{35}$ R. A. Bonham, "Electron impact cross section data for carbon tetrafluoride,” Jpn. J. Appl. Phys., Part 1 33(7S), 4157 (1994).

${ }^{36} \mathrm{~K}$. Nanbu, "Probability theory of electron-molecule, ion-molecule, molecule-molecule, and Coulomb collisions for particle modeling of materials processing plasmas and cases," IEEE Trans. Plasma Sci. 28(3), 971-990 (2000).

${ }^{37}$ K. Nanbu and K. Denpoh, "Monte Carlo collision simulation of positivenegative ion recombination for a given rate constant," J. Phys. Soc. Jpn. 67(4), 1288-1290 (1998).

${ }^{38}$ K. Denpoh and K. Nanbu, "Self-consistent particle simulation of radio frequency $\mathrm{CF}_{4}$ discharge: Effect of gas pressure," Jpn. J. Appl. Phys., Part 1 39(5R), 2804 (2000).

${ }^{39} \mathrm{R}$. Kollath and S. Flügge, Electron emission gas discharge I (Springer, 1956), vol. XXI.

${ }^{40}$ T. Gans, D. O'Connell, V. S. der Gathen, and J. Waskoenig, "The challenge of revealing and tailoring the dynamics of radio-frequency plasmas," Plasma Sources Sci. Technol. 19(3), 034010 (2010).

${ }^{41}$ V. Gedeon, S. Gedeon, V. Lazur, E. Nagy, O. Zatsarinny, and K. Bartschat, "B-spline R-matrix-with-pseudostates calculations for electronimpact excitation and ionization of fluorine," Phys. Rev. A 89(5), 052713 (2014).

${ }^{42}$ R. P. Brinkmann, "Beyond the step model: Approximate expressions for the field in the plasma boundary sheath," J. Appl. Phys. 102(9), 093303 (2007).

${ }^{43}$ J. Schulze, A. Derzsi, K. Dittmann, T. Hemke, J. Meichsner, and Z. Donkó, "Ionization by drift and ambipolar electric fields in electronegative capacitive radio frequency plasmas," Phys. Rev. Lett. 107(27), 275001 (2011).

${ }^{44}$ N. Nakano and T. Makabe, "Influence of driving frequency on narrow-gap reactive-ion etching in SF 6," J. Phys. D: Appl. Phys. 28(1), 31 (1995).

${ }^{45}$ E. Schüngel, I. Korolov, B. Bruneau, A. Derzsi, E. Johnson, T. Lafleur, D. O'Connell, T. Gans, J.-P. Booth, Z. Donkó, and J. Schulze, "Frequency dependence of the symmetry control of electronegative capacitively coupled plasmas by voltage waveform tailoring," J. Phys. D: Appl. Phys. (to be published).

${ }^{46}$ B. Bruneau, M. Lepecq, J. Wang, J. Dornstetter, J. Maurice, and E. V. Johnson, "Effect of ion energy on microcrystalline silicon material and devices: A study using tailored voltage waveforms," IEEE J. Photovoltaics 4(6), 1354-1360 (2014).

${ }^{47}$ B. Bruneau, R. Cariou, J. Dornstetter, M. Lepecq, J. Maurice, P. Roca i Cabarrocas, and E. V. Johnson, "Ion energy threshold in low-temperature silicon epitaxy for thin-film crystalline photovoltaics," IEEE J. Photovoltaics 4(6), 1361-1367 (2014). 